\title{
On-chip near-infrared optical spectrometer based on single-mode Epo waveguide and gold nanoantennas
}

\author{
Maurine Malak, ${ }^{a, \star}$ Konstantins Jefimovs, ${ }^{b, \dagger}$ Irène Philipoussis, ${ }^{a}$ Joab Di Francesco, ${ }^{a}$ and Toralf Scharf ${ }^{a}$ \\ anstitut de Microtechnique, Ecole Polytechnique Fédérale de Lausanne, Optics and Photonics Technology Laboratory, \\ Rue de la Maladière 71B, CH-2002 Neuchâtel, Switzerland \\ ${ }^{b}$ Swiss Federal Laboratories for Material Science and Technology, Laboratory for Electronics/Metrology/Reliability EMPA, \\ Überlandstrasse 129, 8600 Dübendorf, Switzerland
}

\begin{abstract}
We report about optical spectrometry using gold nanostructures printed on top of an integrated optical waveguide. The optical waveguide is a single-mode buried waveguide made from a combination of photo-polymerizable materials and is fabricated by photolithography on a glass substrate. To detect the electric field inside the waveguide, a gold nanocoupler array of thin lines (50 nm thick and $8 \mu \mathrm{m}$ in length) is embedded on top of the aforementioned waveguide. They are produced by e-beam lithography. Both waveguide ports are polished, and the output port, in particular, is coated with a thin gold layer to assimilate a mirror and hence, it enables the creation of stationary waves inside the structure. Stationary waves generated inside the guide constitute a spatial interferogram. Locally, light is out-coupled using the nanocouplers and allows measuring the interferogram structure. The resulting pattern is imaged by a vision system involving an optical microscope with the objective lenses of different magnifications and a digital camera mounted on top of the microscope. The $5 \times$ objective lens demonstrates a superior performance in retrieving the investigated spectrum compared to $20 \times$ and $100 \times$ objectives. Fast Fourier transform is performed on the captured signal to extract the spectral content of the measured signal. () 2014 Society of Photo-Optical Instrumentation Engineers (SPIE) [DOI: 10.1117/1.JMM.13.2.023011]
\end{abstract}

Keywords: polymer waveguide; Epocore/Epoclad; nanoantennas; nanocouplers; field samplers; metallic grating; spectrometer.

Paper 14027P received Mar. 18, 2014; revised manuscript received May 18, 2014; accepted for publication May 21, 2014; published online Jun. 20, 2014

\section{Introduction}

Since its early beginning in $1666,{ }^{1}$ spectroscopy has acquired a large interest due to its numerous applications. ${ }^{2}$ The atomic $^{3}$ and molecular ${ }^{4,5}$ structures are identified by their absorption or emission spectra and from there comes the importance of spectroscopy to the different fundamental sciences: chemistry, physics, and life sciences. Therefore, a complete comprehensive understanding of quantum mechanics, ${ }^{3}$ blackbody radiation theory, atomic structure, and spectra could not be achieved without advancement of spectroscopic studies. Spectroscopic techniques can be classified according to the type of radiative energy involved in the interaction (electromagnetic waves, mechanical waves, or radiation pressure waves). They can also be classified according to the nature of the light-matter interaction occurring during the analysis (absorption, emission, reflection, etc.). Spectroscopy can be achieved using several techniques: ${ }^{6}$ cavity ring down spectroscopy, ${ }^{7}$ cavity enhanced absorption spectroscopy (CEAS), ${ }^{8}$ Raman spectroscopy, ${ }^{9}$ time-resolved spectroscopy, mass spectroscopy, ${ }^{10}$ UV-visible spectroscopy, nuclear magnetic resonance, ${ }^{11}$ and Fourier transform spectroscopy (FTS) ${ }^{12}$ as presented in this research work.

In order to fulfill the increasing demand for spectral analysis, various optical components and microsystems have been conceived. Among others, we can cite: FabryPerot $(\mathrm{FP})^{13}$ and Michelson interferometers (MI), ${ }^{14,15}$ array

\footnotetext{
*Address all correspondence to: Maurine Malak, E-mail: maurine_malak@ yahoo.com

†Present address: Paul Scherrer Institut, 5232 Villigen PSI, Switzerland
}

waveguide grating (AWG), ${ }^{16}$ and standing wave Fourier transform spectrometer. ${ }^{17}$ Recently, a study aiming for miniaturization has been conducted on focal plane array spectrometers. ${ }^{18}$ These devices differ in their architecture, operation principle, fabrication technique, and performance. For example, the FP device presented in Ref. 13 is fabricated by deep reactive ion etching (DRIE) and it consists of curved Bragg reflectors intended to focus the light beam inside the cavity and enhance the quality factor to target CEAS applications. Wavelength scanning, by a tunable laser source, is used to characterize the FP. MI devices presented in Refs. 14 and 15 are also obtained by the same DRIE process in Ref. 13 and they are based on planar Bragg reflector. A regular difference in the optical path difference is used to create a spatial interferogram which is subject to fast Fourier transform (FFT) in order to deduce the source spectrum. The AWG device presented in Ref. 16 is produced by electron beam patterning and plasma etching. Light injected in the input port of the device is analyzed in separate channels at the exit ports of the device.

The physical principles underlying the operation of these devices are quite different. For example, the FP is based on resonance between beams bouncing between two highreflectance mirrors. MI, instead, is a two-beam interference device. The AWG device is an integrated optics device exhibiting a grating behavior. The resolution offered by FP and MI strongly depends on the deployed measurement technique, mainly based on wavelength scanning or spatial scanning and fast Fourier transform (FFT). In contrast, the resolution of the AWG strongly depends on the design 
and the geometrical parameters of the device under consideration. It is worth mentioning that the AWG device has a large size $\left(8 \times 8 \mathrm{~mm}^{2}\right)$ and an elaborated fabrication process is needed opposite to that used for FP and MI which are characterized by their compactness $\left(<1 \mathrm{~mm}^{2}\right)$ and their ease of fabrication.

A device with a wavelength scale has been presented in Ref. 17 in a way to provide further miniaturization for integrated spectrometers. Counter-propagation waves are injected into a silicon waveguide and are detected by nanoscatterers placed on top of the latter. It is based on the principle of stationary-wave integrated Fourier transform spectrometer.

In this research work, we report about the realization of a compact, fully integrated FTS based on micro-nanostructures, combining a micron-sized optical waveguide with an array of nanosamplers. The device is based on the Lippmann configuration ${ }^{17}$ where a spatial interferogram is formed inside the waveguide due to the external mirror embedded at its far end. A metallic grating integrated on top of the device helps in sampling this interferogram to allow the reconstruction of the signal and its spectrum. Compared to the devices mentioned before, the presented device is characterized by its compactness (a few thousands of cubic micrometers) and its fast measurement technique.

\section{Fabrication and Design}

\subsection{Waveguide Fabrication Process}

Epo-materials (provided by Micro Resist Technology $\mathrm{GmbH}$, Berlin, Germany) are used to produce the optical waveguides. Epocore/Epoclad materials were chosen to realize the optical device because they offer unique features compared to other polymeric materials. Among others, they are characterized by high transmittance in the near-infrared range and the possibility of tuning the refractive index. The fabrication process shown in Fig. 1 is deployed for the device realization, and it yields symmetric buried waveguides. As noticed from the schematic, waveguide fabrication is based mainly on photolithography and thin film deposition. For more details about the process steps and parameters, the interested reader can refer to Ref. 19. Details about nanoantennas fabrication and optimization are described in the upcoming sections. It is worth mentioning that an optional black layer can be deposited over the last Epoclad layer. This optional layer will serve as a light absorber that suppresses parasitic stray light as dictated by spectrometry application.

\subsection{Nanoantenna Fabrication Process}

Antenna structures were fabricated by a standard lift-off technique (schematized in Fig. 2) over Epoclad layer deposited on top of the fused silica wafers. In fact, early attempts to manufacture them on the baseline waveguides were unsuccessful mainly because it is difficult to manage nanostructure deposition processes on prestructured surfaces of several micrometer heights. The finally established nanoantennas fabrication process can be described as follows: An Epoclad layer is laid on top of a typical fused silica wafer used as a substrate. Then, a PMMA layer (used as a resist) is deposited on top of the Epoclad. Since fused silica is an insulator, a copper $(\mathrm{Cu})$ conductive layer was added on top of the resist in order to avoid charging during the electron beam

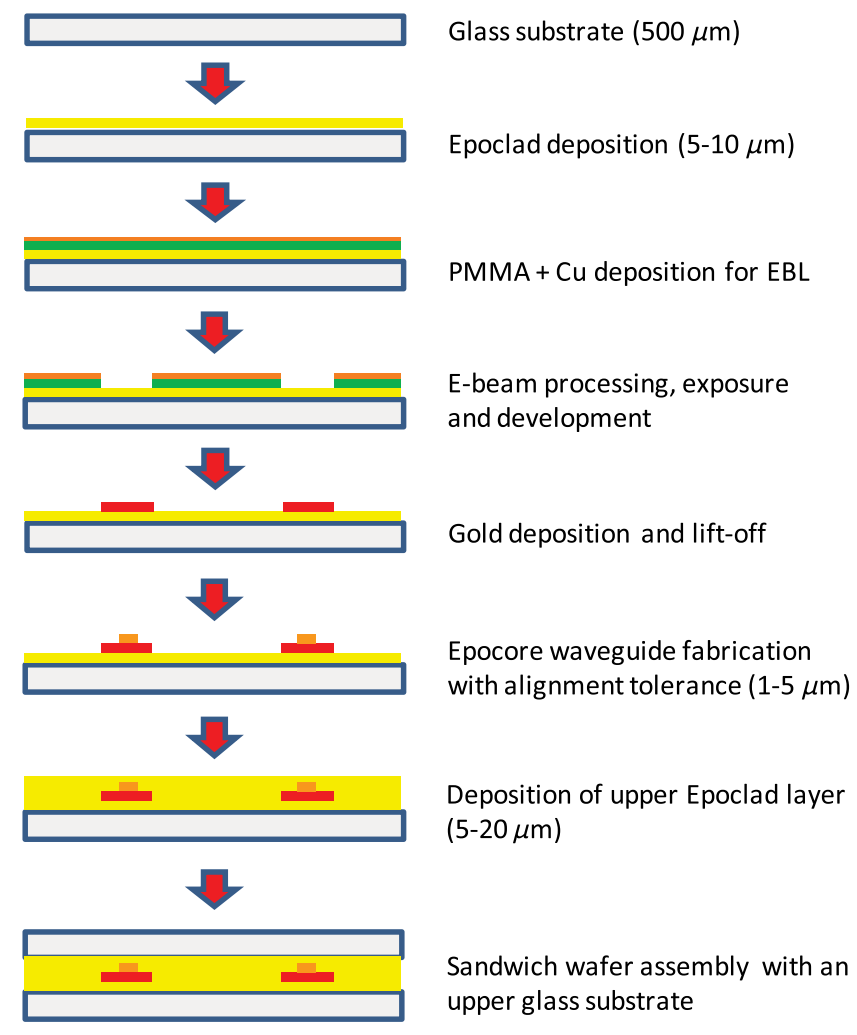

Fig. 1 Schematic illustration of the fabrication process for the sandwich wafer assembly. The drawings highlight the fabrication of the waveguides, the nanoantennas underneath, and the final packaging with the top glass superstrate.

lithography (EBL) step. After EBL exposure, the $\mathrm{Cu}$ layer is removed in weak nitric acid and PMMA is developed in MIBK:IPA solution. After the development, a short oxygen plasma step is applied to clean possible organic residuals in the developed areas of PMMA. Then, the antenna material (typically, gold) is evaporated and then actual lift-off in acetone is performed. So, the metal deposited on top of the resist is removed together with the dissolved resist, while the metal deposited directly onto the substrate surface in the developed areas stays. We can deduce that in order to achieve highest resolution, reproducibility, and quality, the exposure and resist development parameters should be properly adjusted. Also, optimization of the antenna

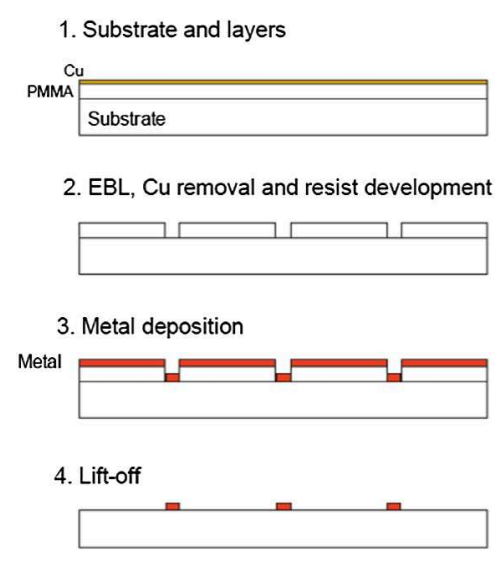

Fig. 2 Schematics of antenna fabrication by electron beam lithography and lift-off. 


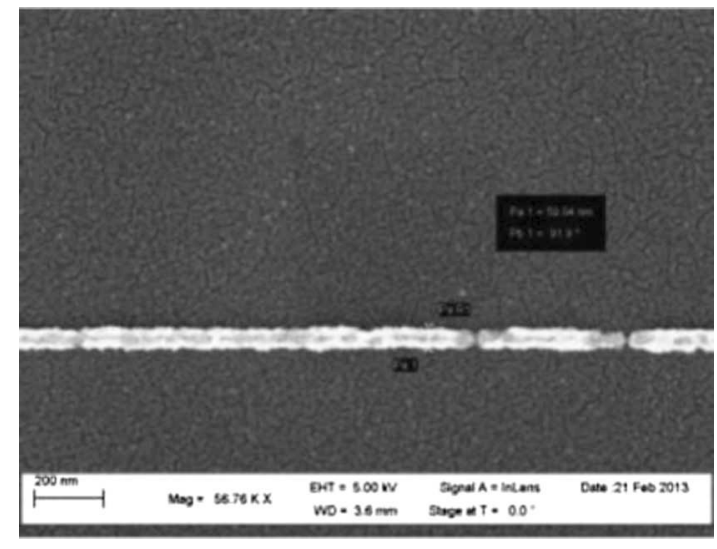

Fig. 3 Examples of antennas fabricated using 5-keV energy. Irregularities are observed in the deposited nanoantennas. The callout indicates a thickness of $59 \mathrm{~nm}$ for a line having a tilt angle of 92 deg.

fabrication process is required at different stages of the sample fabrications and the most important step is the EBL.

\subsection{Optimization of the Antenna Fabrication Parameters}

Our original idea was to limit the EBL energy to $5 \mathrm{keV}$ in order to avoid the so-called proximity effect-that is, exposure of the neighboring area due to scattering of electrons in the substrate. The proximity effect is known to cause the neighboring line broadening and even shifting. However, it is noticed that the minimum linewidth that we could get with $5-\mathrm{keV}$ energy is around $55 \mathrm{~nm}$. This is due to scattering of low-energy electrons in the copper conductive layer deposited on top of the PMMA resist. Although the resolution level is still acceptable, another problem is encountered, which is the lack of quality and reproducibility (Fig. 3). Therefore, we decided to switch to $30-\mathrm{keV}$ energy. Figure 4 shows examples of plasmonic nanoantennas of different lengths fabricated using $30 \mathrm{keV}$. Note that the antennas of different lengths have essentially the same linewidth ( $\sim 4 \mathrm{~nm})$, which means that the proximity effect is negligible. It is found that the structures are very insensitive to dose variations; hence, a change of dose by a factor of two results in a linewidth change of $20 \mathrm{~nm}$ (from 30 to $50 \mathrm{~nm}$ ). Thus, an energy of $30 \mathrm{keV}$ is used as an optimal value for all the future fabrication.

\subsection{Device Design}

The device layout shown in Fig. 5 consists of a micron-sized buried, rectangular optical waveguide on top of which gold nanoantennas acting as field samplers are embedded. The waveguide is realized by photolithography in Epoxy material (Epoclad/Epocore). The core/clad refractive index difference $\Delta n=8.2 \times 10^{-2}$ leads to typical waveguide dimensions of $3 \times 2.2 \mu \mathrm{m}^{2}$, chosen to assure single-mode operation at a wavelength of $784 \mathrm{~nm}$. Nanoantennas, produced by EBL, have dimensions of $50 \mathrm{~nm} \times 20 \mathrm{~nm} \times 8 \mu \mathrm{m}$. They are arranged in a linear array whose period is $2.87 \mu \mathrm{m}$ and overall length is $1.619 \mathrm{~mm}$. The overall device is sandwiched between two glass substrates to preserve it from the environmental conditions. Another step involves polishing the input/ output edges of the chip to provide very smooth interfaces needed for light injection at the device input and for the mirror formation at the output of the device. Finally, a thin gold layer is evaporated at the output interface to integrate the mirror needed to complete the spectrometer structure.

Note that it would have been better to implement smaller periods to improve the resolution of the sampled interferogram and hence the spectral measurement range of the whole device; however, the periodicity of $2.87 \mu \mathrm{m}$ was targeted due to many reasons:

- Smaller periods cannot be distinguished by the implemented vision system because the optical elements (objective lenses) have limited modulation transfer functions.

- The pixel size (resolution) of the camera sensor might not detect scattering from nanostructures of smaller periods due to aliasing.

- The EBL processing time will become tremendous.

So, if the period is increased, the sampled interferogram will become smoother and the spectrum can be retrieved easily without need for extensive numerical processing. Moreover, the spectral range of the instrument will be increased. On the other hand, decreasing the period leads to undersampled interferogram and here, an additional technique should be applied in order to improve the spatial resolution and then derive the signal spectrum. Of course, decreasing the period has many advantages such as relaxed constraints on the choice of the optical elements (lenses) and the camera sensor, reduced EBL drawing time, but on the account of the complex functionality and signal processing.

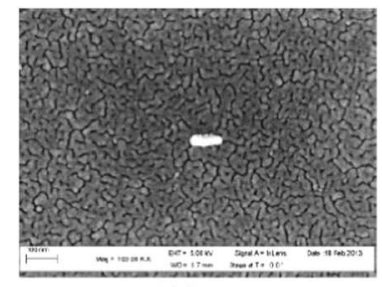

$100 \mathrm{~nm}$

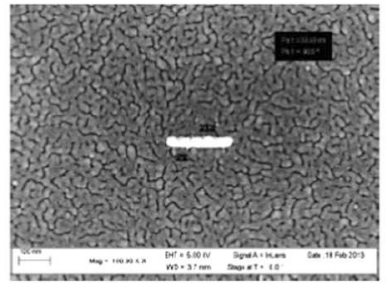

$200 \mathrm{~nm}$

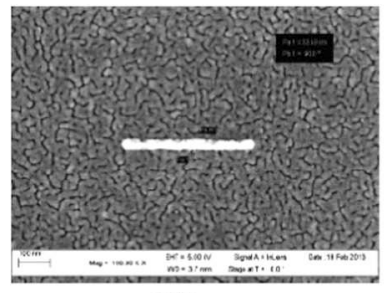

$400 \mathrm{~nm}$

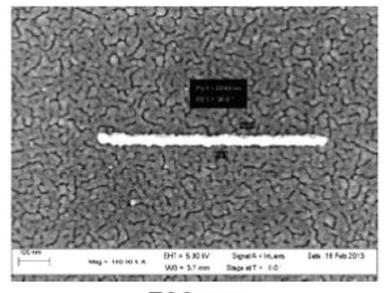

$700 \mathrm{~nm}$

Fig. 4 Examples of antennas fabricated using 30-keV energy. For four different lengths of nanoantennas $(100,200,400$, and $700 \mathrm{~nm})$, we obtain thicknesses of $44 \mathrm{~nm}$ for vertical lines of 90 deg as indicated by the callouts. Higher reproducibility, less defects, and higher tolerance to dose are observed with the beam energy of $30 \mathrm{KeV}$, compared to the case of $5 \mathrm{keV}$. 


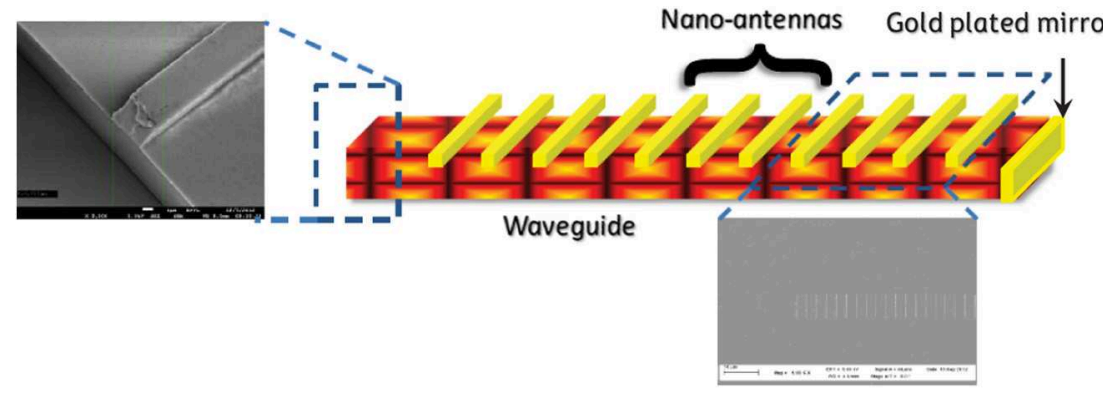

(a)

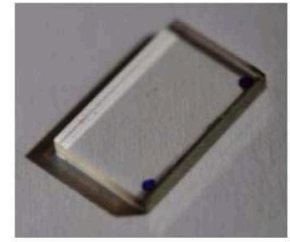

(b)

Fig. 5 (a) Schematic illustration of the device with SEM photos showing the waveguide and the nanoantennas. (b) Chip with a gold mirror on one side. The side, where the gold mirror is located, is indicated by the blue points.

\section{Operation Principle and Analytical Modeling}

The operation principle of the system can be described as follows: light injected into the optical waveguide in the forward direction is back-reflected on itself by the mirror and leads to formation of a standing wave pattern; hence an interferogram is created inside the guide. Here comes the role of the nanoantennas, which act as local field sensors that outcouple a power level corresponding to the spatial light distribution of the interferogram created underneath. The scattered radiation is captured from the top by a microscope equipped with a camera and high-resolution optics. Then, the data are processed to extract the spectral characteristics of the signal and Fourier transform is used to deduce the different spectral components propagating inside the device. It is to be noted that end-fire coupling is used to inject light into the optical waveguide. In addition, the array of nanoantennas may be assimilated to a metallic grating which scatters the standing wave created inside the waveguide toward the far-field.

Despite the similarity between conventional gratings and the suggested metallic grating, the functionality of both gratings is quite different; we would have expected to obtain the spectrum directly at the output of the metallic grating as in conventional gratings. In fact, two basic aspects underlie the difference between the functionality of both devices. In our case, light is guided inside the optical waveguide and its evanescent tail is scattered by the metallic grating, while in conventional gratings free space light is directly incident on the grating. Moreover, light propagates parallel to the metallic grating structure and is sampled by the metallic nanostructures oppositely to conventional gratings where light is projected perpendicular to the grating structure, diffracted, and then analyzed by the grating.

In order to understand the outcome of the Fourier transform result, a simplified analytical model was devised to study the behavior of the lightwave propagating inside the device.

A wave propagating in the forward direction is given by

$E_{\mathrm{fwd}}=E_{0} e^{j \beta z}$,

where $E_{0}$ is the electric field amplitude and $\beta$ is the wave propagation constant, given by $\beta=\frac{2 \pi}{\lambda} n_{\mathrm{eff}}, n_{\mathrm{eff}}$ being the effective refractive index of the mode propagating inside the guide.
Similarly, a wave propagating in the backward direction is given by

$E_{\mathrm{bwd}}=E_{0} e^{-j \beta z}$,

Then, the total field amplitude of the standing wave is given by the sum of the forward and backward waves

$E_{\mathrm{tot}}=E_{\mathrm{fwd}}+E_{\mathrm{bwd}}=E_{0} e^{j \beta z}+e^{i \pi} \cdot e^{-j \beta z}$,

where the phase $\pi$ accounts for the additional phase acquired upon reflection from the mirror.

After mathematical manipulation, we obtain

$\left|E_{\text {tot }}\right|^{2}=2\left|E_{0}\right|^{2}\{1-2 \cos (2 \beta z)\}$.

The previous equation pertains to the envelope of the standing wave pattern. Hence, the spectral content of the sampled signal involves, among others, Fourier components of the injected wavelength $\lambda$ scaled down by $2 n_{\text {eff. It is worth }}$ mentioning that the nanosamplers have a noticeable impact on the signal spectrum. In fact, the nanoantennas introduce, in addition to the components of interest, several side peaks in the spectrum. However, the full analytical model involving the nanoantennas is out-of-scope with respect to this specific case of study and will not be reported in this research paper.

To render this model simple and comprehensive, some basic assumptions have been considered:

1. The mirror is ideal and free from roughness and hence, the wave amplitude is kept unaltered before and after reflections. This assumption reinforces the condition of a scattering-free interface with a reflection phase of $\pi$.

2. The attenuation in the guide is negligible.

\section{Characterization and Measurements}

\subsection{Optical Setup}

The deployed optical setup is illustrated in Fig. 6. Both visible $(658 \mathrm{~nm})$ and infrared $(784 \mathrm{~nm})$ light are used for alignment and measurement purposes, respectively. The infrared light is connected to an optical isolator to suppress any power fed back to the source and then, it assures stability of the emitted laser with a clean mode profile. The visible light, instead, is connected to an optical attenuator to enable 


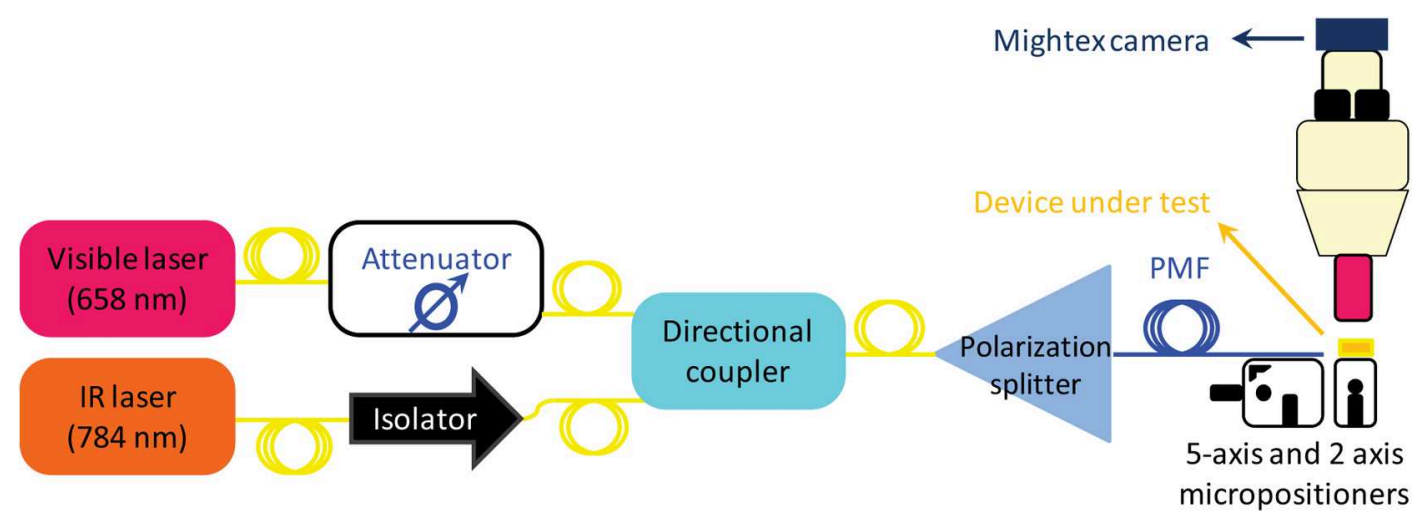

Fig. 6 Schematic representation illustrating the arrangement of the optical components of the setup used to characterize the microspectrometer.

intensity control. The isolator output and the attenuator output are combined by a directional coupler. The coupler output is, in turn, connected to a polarization splitter to divide the polarizations into TE/TM obtained at each output port of the splitter. Finally, a polarization maintaining fiber (PMF) is used at the far end of the setup to transmit only one of the splitted polarizations (TE or TM) to the input of the tested device. The PMF is aligned using Newport five-axis micropositioners, and the device is aligned using Newport two-axis translational micropositioners.

The standing wave pattern generated inside the device is imaged using an optical system involving high-resolution objective lenses having magnifications of $5 \times, 20 \times$, and $100 \times$ (provided by Mitutoyo Corporation, Kawasaki, Japan), a Nikon microscope, and a CMOS camera (provided by Mightex systems, California). The captured photo is treated in MATLAB to extract the spectrum of the injected signal.

\subsection{Optical Measurements}

TE polarization injected into the waveguide generates a stationary wave, and an interference pattern is created. Measurements carried with visible light at $658 \mathrm{~nm}$ and Mitutoyo microscopic objectives $(5 \times, 20 \times$, and $100 \times)$ were used together with Mightex camera to capture standing wave pattern for later analysis with FFT in MATLAB (Fig. 7). Analysis reveals different spectral components according to the deployed objective lens.

With the $100 \times$ objective, we can identify the fundamental component and the harmonics of the nanoantennas spaced by the intervals of $2.87 \mu \mathrm{m}$. Moreover, we can identify two side peaks located at $157.6 \mathrm{~nm}$, close to the DC peak; they pertain to the calibrated pixeling effect of the camera. On the another hand, with the $20 \times$ objective, we can identify other peaks in addition to the nanoantennas component located at $2.887 \mu \mathrm{m}$ and the component of the calibrated pixels located at $0.8 \mu \mathrm{m}$. Those extra peaks arise from the convolution between the aforementioned peaks at $(2.887$ and $0.8 \mu \mathrm{m})$ and they are located at 2.1 and $4.2 \mu \mathrm{m}$. For the $5 \times$ objective, we observe two sets of side peaks. The set located at $232 \mathrm{~nm}$ is mainly due to the laser wavelength $(658 \mathrm{~nm})$ but scaled down by $2 n_{\text {eff }}$ as predicted by the analytical model.

From this analysis, we can say that the injected light can be deduced only when the $5 \times$ objective is used for conducting the measurements because a large field-of-view is covered with the $5 \times$ objective and the captured interferogram involves more wavelength periodicity than with the $20 \times$ or the $100 \times$ objectives. Hence, the source spectral component can be retrieved more easily with the $5 \times$ objective. It is worth mentioning that other peaks are also present in the FFT curves (in the range of 30 to $60 \mathrm{~nm}$ ) due to the geometrical aberrations of the objective lenses. This aberration appears as a smooth envelope superimposed on the image observed in the spatial domain. Upon FFT, it is translated into narrow peaks convoluted with the different wavelength components of the image. For this reason, the 30- to 60-nm peaks are observed close to the DC component and the two side peaks. This effect is more pronounced for the $5 \times$ objective compared to the case of $20 \times$ and $100 \times$. Note that the measurements have been conducted at $783 \mathrm{~nm}$ and the device exhibits similar behavior to that observed at $658 \mathrm{~nm}$.

\subsection{Discussion}

The presented results need careful reconsideration since the measured spectra are heavily undersampled compared to classical FTS where one uses oversampling to recover the measured spectrum in great detail. As noticed, the observation field reduces as the objective lens magnification increases and hence, the number of nanoantennas available for sampling reduces. This limited number of nanoantennas leads to limited spectral resolution and the finite distance between the nanoantennas leads to undersampling. In addition, the different lens magnifications $(5 \times, 20 \times, 100 \times)$ create different spectral resolutions (gradually degrading for high magnification) and different spectral ranges (gradually increasing for high magnification). The FFT range is related to the camera pixel size, number of pixels, and the lens magnification. On the another hand, the resolution is related to the previous set of parameters in addition to the effective number of nanoantennas captured within the field-of-view.

Aside from the device resolution, it is worth mentioning that the spectrometer sensitivity is related to another set of parameters such as the sensitivity of the imaging sensor, the intrinsic losses in Epo material, glass superstrate, air, and the coupling efficiency of the metallic grating. Indeed, rough and discontinuous gold nanostructures will increase the scattering and the losses of the device which will degrade the sensitivity of the microinstrument. 

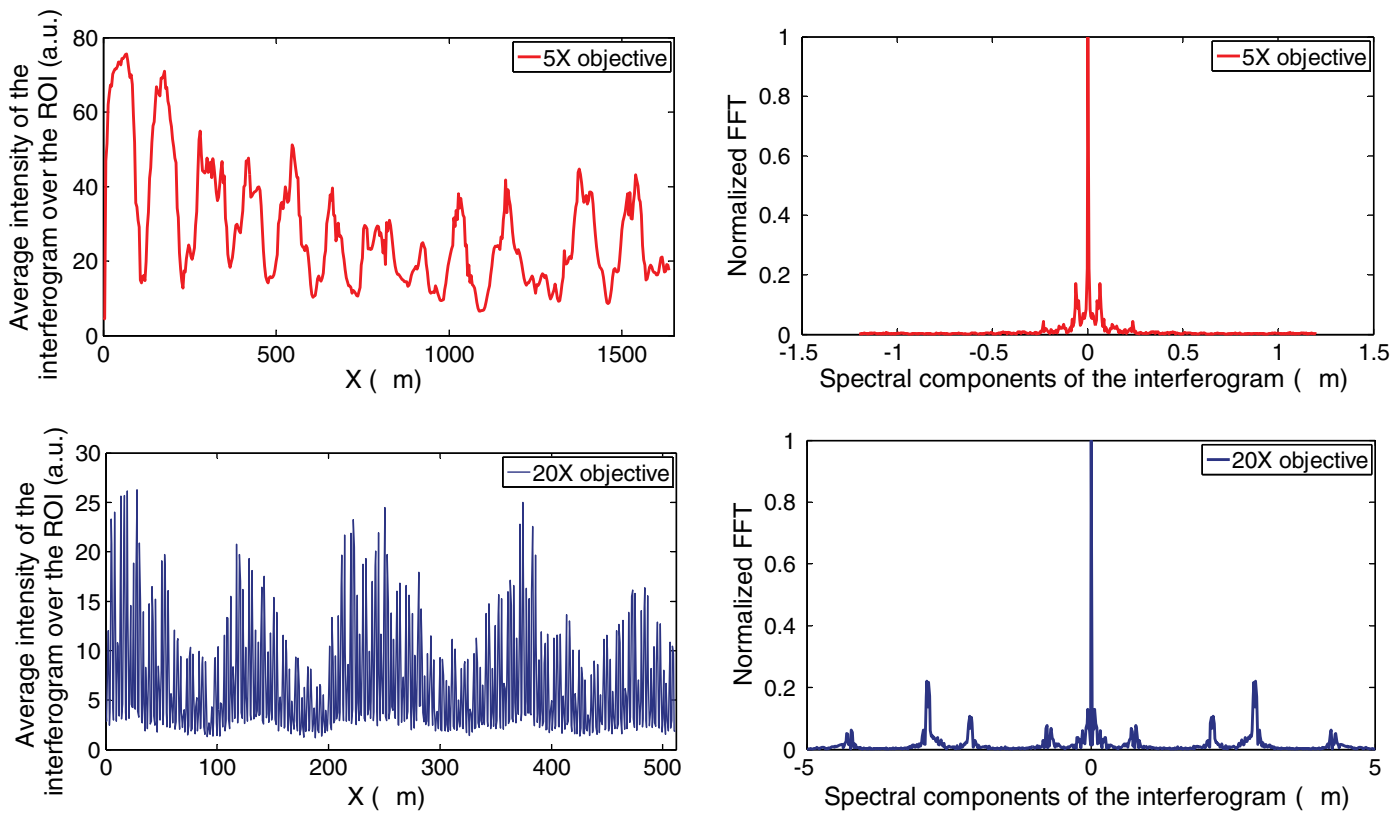

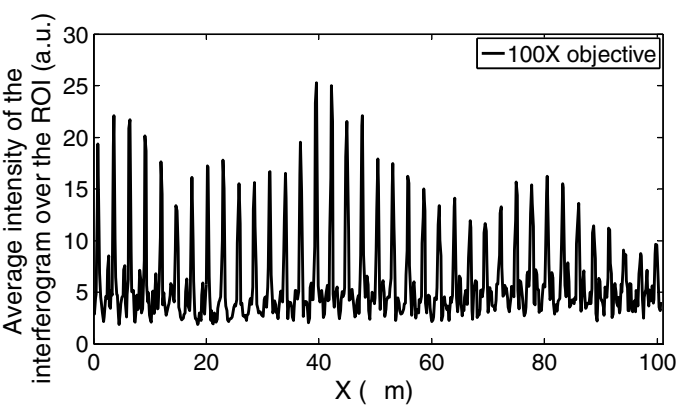

(a)

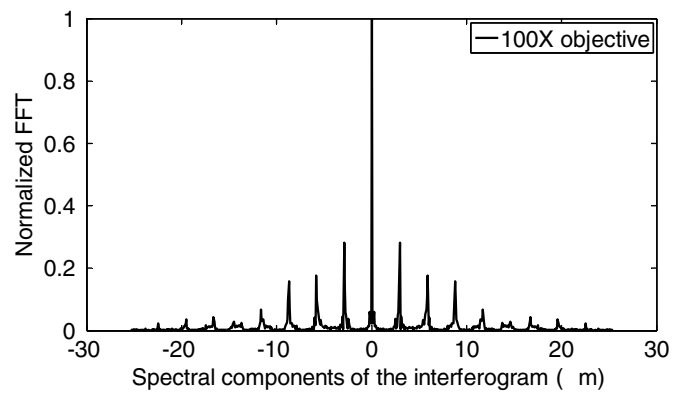

(b)

Fig. 7 (a) Average profile of the measured interferogram over the region of interest for microscopic objectives of $5 \times, 20 \times$, and $100 \times$. (b) Fourier transform of the interferograms in (a).

\section{Conclusion and Perspectives}

To conclude, a miniaturized standing wave spectrometer based on polymer waveguides has been presented. The fabrication process of the micro-nanostructures has been detailed. A simple analytical model that predicts the spectral components content of the injected monochromatic light has been derived. Preliminary experimental results obtained with the $5 \times$ objective elucidate the peaks of the visible laser source. Future work will focus on the development of new designs for increased sampling and easier recovery of the measured spectra.

\section{Acknowledgments}

The authors would like to acknowledge the financial support provided by European Space Agency to achieve this research work.

\section{References}

1. B. R. Masters, "A brief history of spectral analysis and astrospectroscopy," Opt. Photonics News 20(11), 34-39 (2009).

2. S. Chu et al., "Spectroscopy," Article from the Encyclopedia Britannica, August 2008, http://www.britannica.com/EBchecked/topic/558901/ spectroscopy/80590/Applications (December 2013).

3. H. A. Bethe and E. E. Salpeter, Ouantum Mechanics of One- and TwoElectron Atoms, Academic Press, New York (1957).

4. W. Demtröder, Laser Spectroscopy: Basic Concepts and Instrumentation, Springer-Verlag, Berlin (1981).
5. Y. Li, Foundations of Laser Spectroscopy, Wiley \& Sons, California (1984).

6. S. Stenholm, "Spectroscopic techniques," 2009, http://research.pbsci .ucsc.edu/chemistry/li/teaching/chem268/Spectroscopic\%20techniques .pdf (December 2013).

7. M. K. Sprague, "Cavity ringdown spectroscopy, kinetics, and quantum chemistry of atmospherically relevant reactions," PhD Dissertation, California Institute of Technology (2012).

8. M. Malak et al., "Analysis of Fabry-Perot optical micro-cavities based on coating-free all-silicon cylindrical Bragg reflectors," Opt. Express 21(2), 2378-2392 (2013)

9. A. C. Ferrari and D. M. Basko, "Raman spectroscopy as a versatile tool for studying the properties of graphene," Nat. Nanotechnol. 8(4), 235-246 (2013).

10. A. El-Aneed, A. Cohen, and J. Banoub, "Mass spectrometry, review of the basics: electrospray, MALDI, and commonly used mass analyzers," Appl. Spectrosc. Rev. 44, 210-230 (2009).

11. R. W. Darbeau, "Nuclear magnetic resonance (NMR) spectroscopy: a review and a look at its use as a probative tool in deamination chemistry," Appl. Spectrosc. Rev. 41, 401-425 (2006).

12. B. C. Smith, Fundamentals of Fourier Transform Infrared Spectroscopy, CRC Press, Taylor \& Francis Group, Boca Raton, Florida (2011).

13. M. Malak et al., "Micromachined Fabry-Perot resonator combining submillimeter cavity length and high quality factor," Appl. Phys. Lett. 98(21), 211113 (2011)

14. M. Malak et al.,"All-silicon Michelson instrument on chip: distance and surface profile measurement and prospects for visible light spectrometry," Appl. Phys. Lett. 102(14), 141102 (2013).

15. B. Saadany et al., "A miniature Michelson interferometer using vertical Bragg mirrors on SOI," in IEEE/LEOS Int. Conf. on Optical MEMS and their Applications, Big Sky, Montana, pp. 50-51 (2006).

16. P. Cheben et al., "A high-resolution silicon-on-insulator arrayed waveguide grating microspectrometer with sub-micrometer aperture waveguides," Opt. Express 15(5), 2299-2306 (2007). 
17. E. le Coarer et al., "Wavelength-scale stationary-wave integrated Fourier-transform spectrometry," Nat. Photonic 1, 473-478 (2007).

18. B. Guldimann and S. Kraft, "Focal plane array spectrometer: miniaturization effort for space optical instruments," Proc. SPIE 7930, 793000 (2011).

19. M. Malak et al., "Polymer based single mode optical waveguide for spectroscopy applications," Proc. SPIE 8846, 884615 (2013).

Maurine Malak received the BSc and MSc degrees in optics from Ain Shams University (Egypt) in 2004 and 2008, and a PhD degree in optical MEMS from Paris-Est University in 2011. She is a research scientist at EPFL, Switzerland. She has authored more than 30 journal papers and conference proceedings and a book chapter about one-dimensional photonics crystal. She has more than 10 years of experience in multidisciplinary optical subjects. Her research interests include optical MEMS, optofluidics, integrated optics, and microfabrication.

Konstantins Jefimovs received a $\mathrm{PhD}$ degree in physics from University of Joensuu, Finland, in 2003. He is a senior research scientist affiliated with Paul Scherrer Institute (Switzerland). During his career, he mastered nanofabrication and microfabrication technologies. $\mathrm{He}$ is an author of more than 50 papers in peer-reviewed international journals. His main research interests include development of fabrication techniques for various applications, including photonics, plasmonics, metamaterials, diffractive optics, $x$-ray microscopy, grating based $x$-ray, and neutron-based interferometry.
Irène Philipoussis received a federal certificate for laboratory assistantship in physics from CERN, Switzerland, in 1986. She is a technician at EPFL. From 1986 to 1990 , she was with the research department of Oscilloquartz working on the development of atomic clocks. From 1990 to 1997, she was in Asulab working on the development of LCD. Since 1997, she has been with EPFL. Her research interests include fabrication and development of diffractive/refractive micro-optical components for imaging applications.

Joab Di Francesco received the engineering diploma from the University of Applied Sciences (HE-ARC), Switzerland, in 2009. He is a research engineer at EPFL. From 2009 to 2011, he was with the Frequency and Time Laboratory of Neuchâtel University, Switzerland. He worked on development and testing of frequency stabilized VCSEL locked to microfabricated Rb cell, realization of mid-IR quantum cascade detector. His research interests include spectroscopy and conception of optical devices for the mid-IR region.

Toralf Scharf received a MSc degree in surface physics from University of Duisburg (Germany) in 1993 and a PhD degree from University of Halle (Germany). He is a senior scientist with EPFL. $\mathrm{He}$ focuses his research activities on interdisciplinary subjects bringing together microsystem, material technology, and optics. $\mathrm{He}$ has extensive experience of more than 15 years in optics. His research activities are quite broad, ranging from liquid crystal optics to amorphous nanophotonics. 